Mahmoudvand et al., Afr J Tradit Complement Altern Med., (2017) 14 (5): 49-55

https://doi.org/10.21010/ajtcam.v14i5.7

\title{
CHEMICAL COMPOSITION AND PROPHYLACTIC EFFECTS OF SATURJA KHUZESTANICA ESSENTIAL OIL ON ACUTE TOXOPLASMOSIS IN MICE
}

\section{Hossein Mahmoudvand ${ }^{1}$, Maryam Beyranvand ${ }^{2}$, Hassan Nayebzadeh ${ }^{3}$, Shirzad Fallahi ${ }^{1,4 *}$, Seyyed Reza Mirbadie $^{5}$, Farnaz Kheirandish ${ }^{1,4}$, Mohammad Hassan Kayedi ${ }^{4}$}

${ }^{1}$ Razi Herbal Medicines Research Center, Lorestan University of Medical Science, Khorramabad, Iran ${ }^{2}$ Department of Parasitology, Faculty of Veterinary, Borujerd Branch, Islamic Azad University, Borujerd, Iran., ${ }^{3}$ Department of Pathobiology, Faculty of Veterinary Medicine, Lorestan University, Khorramabad, Iran. ${ }^{4}$ Department of Medical Parasitology and Mycology, Faculty of Medicine, Lorestan University of Medical Sciences, Khorramabad, Iran. ${ }^{5}$ School of Medicine, Shahroud University of Medical Sciences, Shahroud, Iran.

* Corresponding Author E-mail: Shfupdate@gmail.com, Falahi.Sh@lums.ac.ir

\begin{tabular}{|c|}
\hline \multicolumn{1}{|c|}{ Article History } \\
Received: 29, Sept. 2016 \\
Revised Received: 01, Dec. 2016 \\
Accepted: 06, Dec. 2016 \\
Published Online: 01, Oct. 2017 \\
\hline
\end{tabular}

Abstract

Background: Toxoplasma gondii is a widespread zoonotic protozoan that infects approximately one third of the global human population and all other warm-blooded animals. The present study aims to evaluate the prophylactic effects of Satureja khuzestanica essential oil (SKEO) on infected mice with acute toxoplasmosis.

Materials and Methods: The components of the SKEO were identified by gas chromatography/mass spectroscopy (GC/MS). To evaluate the prophylactic effects of SKEO, mice were divided into four groups. (i) non-treated group, (ii) mice treated with olive oil once a day for two weeks, (iii) mice treated with SKEO at the dose of $0.2 \mathrm{ml} / \mathrm{kg}$ once a day for two weeks, (iv) and mice orally treated with SKEO at the dose of $0.3 \mathrm{ml} / \mathrm{kg}$ once a day for two weeks. After $24 \mathrm{~h}$ (fifteenth day) mice in the groups of two-four were infected intraperitonealy with $10^{-4}$ tachyzoite of $T$. gondii, RH strain. The mortality rate in all infected mice and the number of tachyzoites from infected mice were recorded.

Results: The main components of SKEO were carvacrol $(78.8 \%)$, thymol $(7.5 \%)$, and beta-Bisabolene (1.2\%). Findings of prophylactic effects revealed that mortality rate of infected mice was 8 days after oral administration of SKEO at the concentration of 0.2 and $0.3 \mathrm{ml} / \mathrm{kg}(\mathrm{P}<0.05)$. In contrast, this value for control group was 5 days. In addition, SKEO significantly reduced the mean number of tachyzoites compared with control group $(\mathrm{P}<0.05)$. No significant difference $(\mathrm{P}>0.05)$ was observed in the clinical chemistry and hematological parameters following oral administrations of SKEO at the doses of 0.2 and $0.3 \mathrm{ml} / \mathrm{kg}$ for 14 days.

Conclusion: The results showed the potential of SKEO as a natural source for the production of new prophylactic agent for use in toxoplasmosis.

Keywords: GC/MS; Satureja khuzestanica; Toxoplasma gondii; In vivo; Mice

\section{Introduction}

Toxoplasma gondii as an intracellular protozoan parasite can infect a broad spectrum of warm-blooded animals as well as humans (Hill and Dubey 2002). Human infection to toxoplasmosis occurs through three ways: eating oocysts with food, eating raw meat containing tissue cysts and also from mother to fetus (Robert-Gangneux and Darde 2012; Mahmoudvand et al., 2016a). Although, toxoplasmosis cause no symptoms in healthy humans; but in immunocompromised individuals, for example persons with HIV/AIDS, receiving an organ transplant, toxoplasmosis may result in severe manifestations, such as myocarditis, and encephalitis (Derouin and Pelloux 2008; Signorini et al., 2007). To date, chemotherapy of $T$. gondii with the combination of pyrimethamine and sulfadiazine, plus folic acid, is the preferred option to treat toxoplasmosis (Montoya and Liesenfeld 2004). Reviews have reported that side-effects of pyrimethamine are: osteoporosis, liver toxicity, as well as teratogenic effects, particularly in patients with immune deficiency syndromes (Georgiev 1994; Parsil 2009). Sulfadiazine, a sulfonamide antibiotic, is also applied along with pyrimethamine to treat $T$. gondii infection. However, some adverse side-effects, including faintness, lethargy, headache, and vomiting, limit its use (Georgiev 1994; Parsil 2009). 
The use of plant medicines is typically widespread to treat a number of diseases such as infectious ones as well as keep public health (Rocha et al., 2005; Ezatpour et al., 2015). Since the last few decades, natural products have played a key role in the search for new drugs to treat a variety of human diseases (Cowan 1999). Satureja khuzestanica Jamzad is a medicinal plant which commonly grows in Western Iran (Hadian et al., 2011). In traditional medicine, $S$. khuzestanica has been widely used as an algesic, anti-diarrhea, and antiseptic (Hajhashemi et al., 2010; Hadian et al., 2011). Moreover, recent studies have reported several therapeutic properties of the plant, such as antioxidant, antiinflammatory, hepatoprotective and antimicrobial properties (Amanlou et al., 2004; Ghazanfari et al., 2006; Sanchez de Rojas et al., 1999; Sadeghi-Nejad et al., 2010; and Safarnavadeh et al., 2011). Previous studies have demonstrated that the principal components of the S. khuzestanica essential oil (SKEO) in greatest species are to be phenols, carvacrol, thymol, $p$-cymene, $\beta$-caryo - phyllene, linalool, monoterpenes, sesquiterpenes, and alcohols (Farsam et al., 2004; Hadian et al., 2011). However, chemical composition of essential oils is depended on some agents such as the geographical origin of the variety and collection time (Saedi Dezaki et al., 2015; Yesil Celiktas et al., 2007). Here we aimed to access the chemical composition and prophylactic effects of SKEO in mice model of acute toxoplasmosis.

\section{Materials and Methods Plant materials}

Plant materials (aerial parts) of $S$. khuzestanica were collected from rural regions of Lorestan province, West of Iran, in May 2015. They were identified by a botanist of the Botany Department of Lorestan University, Lorestan, Iran. Voucher specimens have been deposited in the herbarium of Research Center for Agriculture Sciences, Lorestan University of Medical Sciences, Khorramabad, Iran (LF 2522).

\section{Isolation of essential oil}

Room air-dried plant sample was subjected to hydro-distillation for $3 \mathrm{~h}$ using an all-glass Clevenger type apparatus. The obtained essential oil was dried over anhydrous sodium sulfate and stored in darkness at $4^{\circ} \mathrm{C}$ until testing (Mahmoudvand et al., 2016c).

\section{Gas chromatography/mass spectrometry (GC/MS) analysis of essential oil GC analysis}

GC analysis was carried out by Hewlett-Packard 6890 (Hewlett-Packard, Palo Alto, CA) with a HP-5MS column $(30 \mathrm{~m} \times 0.25 \mathrm{~mm}$, film thickness $0.25 \mathrm{~mm})$. The column temperature was maintained at $55^{\circ} \mathrm{C}$ for $3 \mathrm{~min}$, programmed to $180^{\circ} \mathrm{C}$ in the rate of $5^{\circ} \mathrm{C}$ per min, and kept constant at $220^{\circ} \mathrm{C}$ for 5 min. Injector and interface temperatures were 220 and $290^{\circ} \mathrm{C}$, respectively. The flow rate of helium as carrier gas was $(1 \mathrm{~mL} / \mathrm{min} \mathrm{C.F})$. The percentages were calculated by the electronic integration of FID peak areas without using response factor correction. Linear retention indices for all the components were determined by the co-injection of the samples with a solution containing homologous series of C8-C22 n-alkanes.

\section{GC/MS analysis}

GC/MS analysis was performed using a Thermoquest-Finnigan gas chromatograph equipped with fused silica capillary DB-5 column $(30 \mathrm{~m} \times 0.25 \mathrm{~mm}$, film thickness $0.25 \mathrm{~mm})$ coupled with a TRACE mass (CAS, Manchester, UK). Helium was used as the carrier gas with an ionization voltage of $70 \mathrm{eV}$. Ion source and interface temperatures were 220 and $290^{\circ} \mathrm{C}$, respectively. Mass range was from 40 to $400 \mathrm{u}$. Temperature program of the oven was as mentioned above for the GC.

\section{Identifying the essential oil components}

The components of the essential oils were determined by evaluating their relative retention time and mass spectra with the standards, Wiley 2001 library data of the GC/MS system, or those reported in the literature data (Adams 2004).

\section{Parasite}

T. gondii (RH strain) tachyzoites was kindly obtained from the Parasitology Laboratory at the Department of Parasitology and Mycology, Tehran University of Medical Sciences, Tehran, Iran. Parasites $\left(1 \times 10^{4}\right)$ were inoculated in the mice, and after $72 \mathrm{~h}$, tachyzoites were provided by repeated flushing of the peritoneal cavity by Phosphate Buffered Saline (PBS). Tachyzoites were then harvested and recovered with PBS and used in the experiments. To establish animal model of acute toxoplasmosis each group of mice was inoculated intraperitonealy (IP) with $1 \times 10^{4}$ tachyzoite of T. gondii. 


\section{Animals}

Forty-eight male NMRI mice (6-8 weeks old) weighing from 20 to $25 \mathrm{~g}$ were obtained from the Pasteur Institute, Tehran, Iran. Animals were housed in a colony room with a $12: 12 \mathrm{~h}$ light/ dark cycle at $21 \pm 2{ }^{\circ} \mathrm{C}$ and were handled according to standard protocols for the use of laboratory animals.

\section{Prophylactic effects of SKEO}

To evaluate the prophylactic effects of SKEO, mice were divided into four groups (of 12 mice per each group). (i) non-treated group, (ii) mice treated with olive oil once a day for two weeks, (iii) mice treated with SKEO at the dose of $0.2 \mathrm{ml} / \mathrm{kg}$ once a day for two weeks, (iv) and mice orally treated with SKEO at the dose of $0.3 \mathrm{ml} / \mathrm{kg}$ once a day for two weeks. After 24h. (fifteenth day) mice in the groups of two-four were infected intraperitonealy with $10^{4}$ tachyzoite of $T$. gondii, RH strain. Then, the following parameters were performed to determine the prophylactic effects of SKEO against acute toxoplasmosis induced by RH strain of T. gondii in mice model.

\section{Mortality rate}

The mortality rate was determined according to the following formula:

$$
\mathrm{MR}=\frac{\text { Number of dead mice }}{\text { Number of mice at the beginning of the experiment }} \times 100
$$

\section{Parasite burden}

To evaluate the parasite burden in the infected mice, the mean number of $T$. gondii tachyzoites in the peritoneal fluid of mice were calculated in 10 high power field (HPF) and then the mean number of tachyzoites 10/HPF was estimated. Finally, the mean number of tachyzoites in each subgroup of infected mice was calculated (Tavakoli Kareshk et al., 2015).

\section{Ethical statement}

The present investigation was performed in line with the National Institutes of Health. The present study was admired by the Committee on the Ethics of Animal Experiments of the Lorestan University of Medical Science, Iran (no. 93/110).

\section{Statistical analysis}

Data analysis was carried out using SPSS statistical package version 17.0 (SPSS Inc., Chicago, IL, USA). One-way ANOVA with Tukey's post-hoc test was used to assess differences between experimental groups. In addition, $P<0.05$ was considered statistically significant.

\section{Results}

\section{GC/MS analysis of SKEO}

Table 1 shows the findings by GC/MS analysis of SKEO. Sixteen compounds were identified, indicating $99.6 \%$ of the total oil. The main components were carvacrol $(78.8 \%)$, thymol $(7.5 \%)$, and beta-bisabolene (1.2\%) (Table 1).

\section{Prophylactic effects of SKEO}

Figure 1 shows the mortality rate of infected mice in tested groups. The mortality rate of mice in control group was $100 \%$ in the fifth day. Moreover, $100 \%$ mortality was observed in the $7^{\text {th }}$ and $8^{\text {th }}$ days after oral administration of SKEO at the concentrations of 0.2 and $0.3 \mathrm{ml} / \mathrm{kg}$, respectively; which demonstrated that SKEO significantly increased the time of death in the infected mice by acute toxoplasmosis (Fig. 1).

The results obtained demonstrated that the mean number of tachyzoites was $260 \times 10^{4}$ and $189 \times 10^{4}$ for infected mice treated with 0.2 and $0.3 \mathrm{ml} / \mathrm{kg}$ respectively, while in control group, the mean number of tachyzoites was $390 \times 10^{4}$ parasite. The results show that the difference in mortality rate between the SKEO at the concentration of 0.2 and $0.3 \mathrm{ml} / \mathrm{kg}$ and the control group was statistically significant $(\mathrm{P}<0.05)$. The difference in mean number of tachyzoites between infected mice received SKEO and the control group was also statistically significant $(P<0.05)$ (Fig. 2). 
Table 1. Essential oil composition of S. khuzestanica identified by GC-MS

\begin{tabular}{cccc}
\hline No & Components & RT & \% Composition \\
\hline 1. & beta-Myrcene & 9.47 & 0.2 \\
2. & 1 -Phellandrene & 9.89 & 0.2 \\
3. & $\alpha$-Terpinene & 10.31 & 0.2 \\
4. & $\rho$-Cymene & 10.41 & 0.8 \\
5. & Limonene & 10.73 & 0.2 \\
6. & $\gamma$ - Terpinene & 11.69 & 0.3 \\
7. & $\alpha$ - Terpineolene & 12.67 & 0.2 \\
8. & Linalool & 12.88 & 0.5 \\
9. & Borneol & 14.85 & 0.5 \\
10. & Terpinene-4-ol & 15.27 & 0.9 \\
11. & $\alpha$ - Terpineolene & 15.86 & 0.3 \\
12. & Thymol & 18.53 & 7.5 \\
13. & Carvacrol & 18.96 & 78.8 \\
14. & Carvone & 19.16 & 7.6 \\
15. & beta.-Bisabolene & 24.57 & 1.2 \\
16. & Cis-.Alpha.-Bisabolene & 25.32 & 0.2 \\
& Total & & 99.6 \\
\hline
\end{tabular}

${ }^{\mathrm{a}}$ Retention time

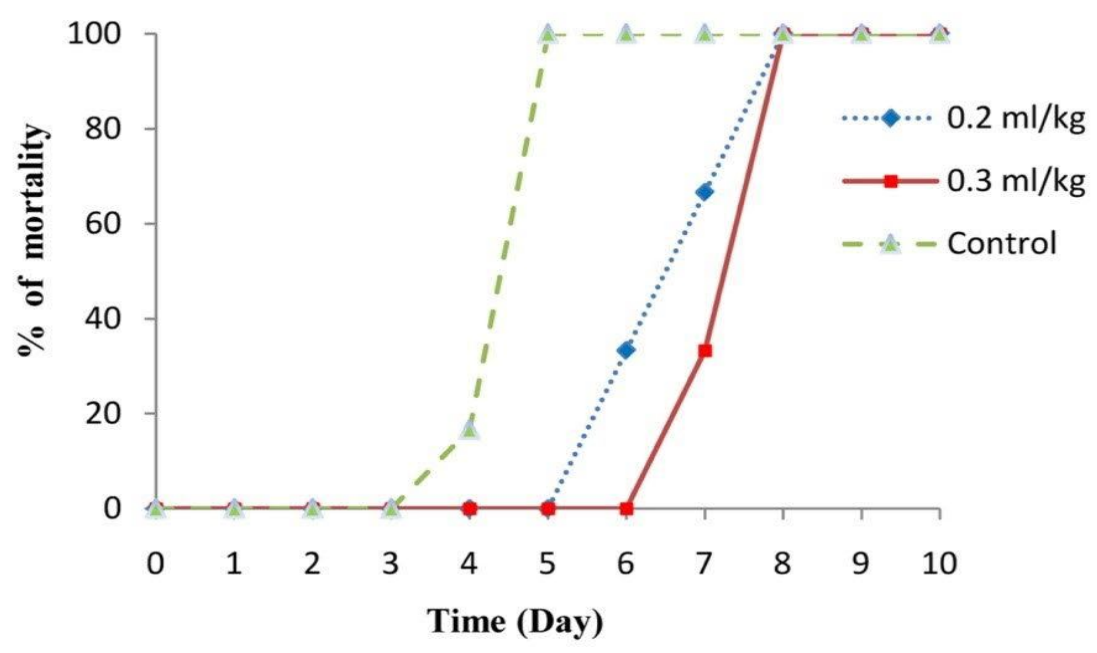

Figure 1: Prophylactic effects of Satureja khuzistanica essential oil on the mortality rate of infected mice with acute toxoplasmosis 


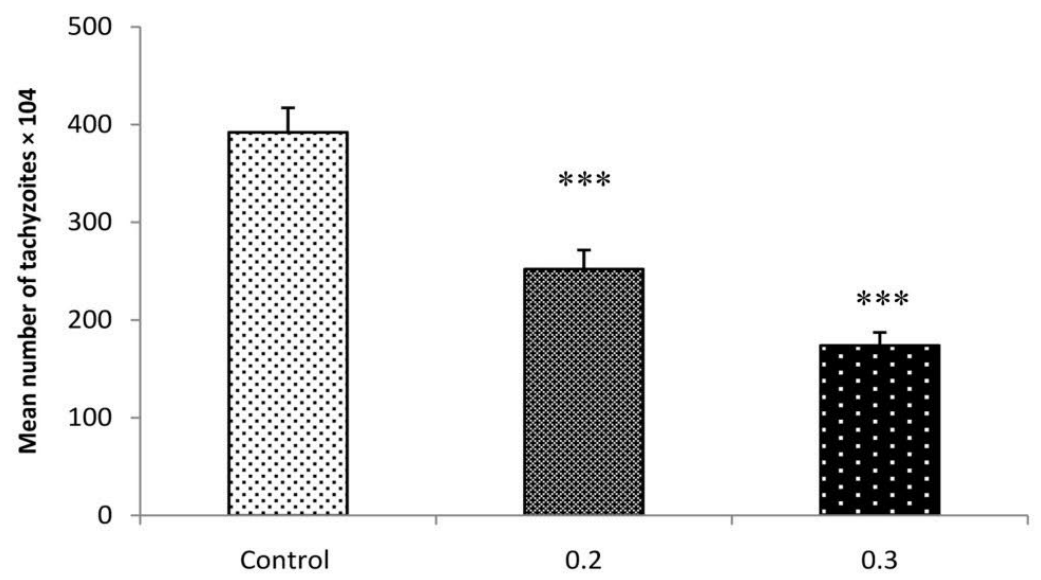

Figure 2: Effects of various concentrations of Satureja khuzistanica essential oil on the mean number of tachyzoites of infected mice with acute toxoplasmosis

\section{Discussion}

Based on the World Health Organization (WHO) reports, more than half of the world used herbal medicine for their early medical supplies. Traditionally, humans trust in plant medicines because of possessing high efficacy, low toxicity and high availability for treatment of a wide spectrum of diseases such as infections ones (Rocha et al., 2005). The present investigation was designed to explore the prophylactic activity on infected mice with acute toxoplasmosis.

According to the obtained findings, oral administration of SKEO for two weeks at the doses of 0.2 and 0.3 $\mathrm{ml} / \mathrm{kg}$ exhibited considerable prophylactic effects against acute toxoplasmosis in mice. The results also revealed that SKEO at the doses of 0.2 and $0.3 \mathrm{ml} / \mathrm{kg}$ significantly decreased the mean of tachyzoites burden in infected mice. Regarding prophylactic effects of herbal medicines, Tavakoli Karesh et al (2015) have demonstrated the capability of Bunium persicum essential oil as a possible resource for the development of new prophylactic drug for toxoplasmosis. Previous studies have reported antiparasitic, antibacterial and antifungal effects of S. khuzestanica. Some researchers also demonstrated the antifungal effect of $S$. khuzestanicaon against several fungal pathogenic strains (Sadeghi-Nejad et al., 2010). Zomorodian et al. (2015) have reported potent antimicrobial effects of SKEO against pathogens causing oral infections including Candida spp. Staphylococcus spp. and Entrococcus spp. in in vitro models. Regarding antiparasitic effects of $S$. khuzestanica, in a study conducted by Kheirandish et al (2011) it has been reported that SKEO significantly improved cutaneous leishmaniasis in BALB/c mice infected by Leishmania major. Moreover, Moazeni et al (2012) have studied the scolicidal effect of S. khuzistanica essential oil on Echinococcus granulosus protoscoleces; they demonstrated that $S$. khuzistanica essential oil has a large amount of carvacrol, and that it can be considered as a proper scolicidal agent.

In this study, we found that the main components of SKEO are carvacrol (78.8\%), thymol (7.5\%), and betaBisabolene (1.2\%), respectively. Consistent with our study, Kheirandish et al. (2011) and Farsam et al. (2004) have demonstrated that the main component of SKEO is carvacrol. However, it should be mentioned that chemical composition essential oil is related to species, weather, collection time, and morphological parts used (Saedi Dezaki et al., 2015; Yesil Celiktas et al., 2007). Carvacrol is a monoterpenoid phenol which possesses antiseptic, antibacterial, antifungal, as well as anti-nociceptive and anti-inflammatory properties (Hajhashemi et al., 2002; Ultee et al., 2002). Additionally, Monzote et al. (2014), revealed that carvacrol had significant leishmanicidal activity with the $\mathrm{IC}_{50}$ value of $15.3 \mathrm{mg} / \mathrm{mL}$ for promastigotes, and $13.6 \mathrm{mg} / \mathrm{mL}$ for the amastigote forms of L. amazonensis. Thus, phytoconstituents of SKEO might be answerable for its prophylactic effects, although their precise method of action is not entirely clear. However, regarding the mechanism of action of phenolic compounds, such as carvacrol and thymol, it has been shown that these compounds are proton exchangers, thus dropping the gradient through the cytoplasmic wall. They cause break down of proton-motrice force and reduction of the ATP pool, leading finally to cell death (Arfa et al., 2006).

In the case of toxicity effects of S. khuzistanica, Abdollahi et al. (2003) demonstrated that oral administration of $S$. khuzistanica essential oil to rats produced a noticeable antioxidant, antidiabetic, antihyperlipidemic effects with no severe complications. The obtained results of the current study revealed the considerable prophylactic efficacy of the SKEO in mice model of acute $T$. gondii infection. Moreover, these outcomes offer the scientific confirmation that natural plants could be applied in traditional remedy to prevent and treat parasitic infections. Nevertheless, additional investigations are required to assess the precise effects of SKEO and its immunomodulatory effect on acute toxoplasmosis.

\section{Acknowledgment}

This study was supported by Herbal and Traditional Medicine Research Center and Vice Chancellor for Research, Kerman University of Medical Sciences (project no. 93/110). The authors would like to thank Ms. Zeinivand 
for data collection and in vivo experiments.

Conflict of Interest: Authors declare that there is no conflict of interest in this study.

\section{References}

1. Abdollahi M, Salehnia A, Mortazavi HR, Ebrahimi M, Shafiee A, Fouladian F, Keshavarz K, Kazemi A. (2003). Antioxidant, antidiabetic, antihyperlipidemic, reproduction stimulatory properties and safety of essential oil of Satureja khuzistanica in rat in vivo: a toxicopharmacological study. Med Sci Monit. 9:331-335.

2. Adams RP. (2004). Identification of essential oil components by gas chromatography/mass spectroscopy. Carol Stream, IL: Allured Publishing Corporation.

3. Amanlou M, Fazeli MR, Arvin A (2004). Antimicrobial activity of crude methanolic extract of Satureja khuzistanica. Fitoterapia. 75:768-770.

4. Araujo FG, Remington JS (1974). Effect of clindamycin on acute and chronic toxoplasmosis in mice. Antimicrob Agents Chemother. 5:647-651.

5. Arfa AB, Combes S, Preziosi-Belloy L, Gontard N, Chalier P (2006). Antimicrobial activity of carvacrol related to its chemical structure. Letters Appl. Microbiol. 43:149-154.

6. Cowan MM. (1999). Plant products as antimicrobial agents. Clin Microb Rev 12:564-82.

7. Derouin F, Pelloux H (2008). Prevention of toxoplasmosis in transplant patients. Clin Microbiol Infect. 14: 1089-1101.

8. Ezatpour B, Saedi Dezaki E, Mahmoudvand H, Azadpour M, Ezzatkhah F (2015). In vitro and in vivo antileishmanial effects of Pistacia khinjuk against Leishmania tropica and Leishmania major. Evid Based Complement Alternat Med. 149707.

9. Farsam H, Amanlou M, Radpour MR, Salehinia AN, Shafiee A (2004). Composition of the essential oils of wild and cultivated Satureja khuzistanica Jamzad from Iran. Flavour Frag. J. 19:308-310.

10. Georgiev VS (1994). Management of toxoplasmosis. Drugs. 48:179-188.

11. Ghazanfari G, Minaie B, Yasa N, AshtaralNakhai L, Mohammadirad A, Nikfar S, Dehghan G, Boushehri VS, Jamshidi H, Khorasani R. Salehnia A, Abdollahi M (2006). Biochemical and histopathological evidences for beneficial effects of Satureja khuzistanica Jamzad essential oil on the mouse model of inflammatory bowel diseases. Toxicol Mech Meth. 16:365-372.

12. Hadian J, Mirjalili MH, Kanani MR, Salehnia A, Ganjipoor P (2011). Phytochemical and morphological characterization of Satureja khuzistanica Jamzad pop-ulations from Iran. Chem. Biodivers. 8:902-915.

13. Hajhashemi V, Ghannadi A, Pezeshkian SK (2002). Antinocicieptive and anti-inflammatory effects of Saturej ahortensis L. extracts and essential oil. J Ethnopharmacol. 82:83-87.

14. Hajhashemi V, Sadraei H, Ghannadi AR, Mohseni M (2010). Antispasmodic and antidiarrhoeal effect of Satureja hortensis L. J Ethnopharmacol. 3:36-40.

15. Hill D, Dubey J (2002). Toxoplasma gondii: transmission, diagnosis and prevention. Clin Microb Infect 8(10): 634640.

16. Kheirandish F, Delfan B, Farhadi S, Ezatpour B, Khamesipour A, Kazemi B, Ebrahimzade F, Rashidipour M (2011). The effect of Satureja khuzestanica essential oil on the lesions induced by Leishmania major in BALB/c mice. African J Pharm Pharmacol. 5(5): 648-653.

17. Mahmoudvand H, Ziaali N, Ghazvini H, Shojaee S, Keshavarz H, Esmaeeilpour K, Sheibani V (2016a). Toxoplasma gondii infection promotes neuroinflamation through cytokine networks and induced hyperalgesia in BALB/c mice. Inflamation. 39(1), 405-412.

18. Mahmoudvand H, Kheirandish F, Saedi Dezaki E, Shamsaddini S and Fasihi Harandi M (2016b). Chemical composition, efficacy and safety of Pistacia vera (var. Fandoghi) to inactivate protoscoleces during hydatid cyst surgery. Biomed Pharmacother 82: 393-398.

19. Mahmoudvand H, Mirbadie SR, Sadooghian S, Fasihi Harandi M, Jahanbakhsh S, Saedi Dezaki E (2017). Chemical composition and scolicidal activity of Zataria multiflora Boiss essential oil. J Essential Oil Res; 29 (1): 42-47.

20. Moazeni M, Saharkhiz MJ, Hoseini AA, MootabiAlavi A. In vitro scolicidal effect of Satureja khuzistanica (Jamzad) essential oil (2012). Asian Pac J Trop Biomed. 2(8): 616-620.

21. Montoya JG, Liesenfeld O (2004). Toxoplasmosis. Lancet 363 (9425):1965-1976.

22. Monzote L, Garci'a M, Pastor J, Gill L, Scull R, Maes L, Gille L. (2014). Essential oil from Chenopodium ambrosioides and main components: Activity against Leishmania, their mitochondria and other microorganisms. Exp Parasitol 136:20-26.

23. Prásil P (2009). Current options for the diagnosis and therapy of toxoplasmosis in HIV-negative patients. Klin Mikrobiol Infekc Lek. 15(3):83-90.

24. Robert-Gangneux F, Darde ML (2012). Epidemiology of and diagnostic strategies for toxoplasmosis. Clin Microbiol Rev 25: 264-296.

25. Rocha LG, Almeida JR, Macedo RO, Barbosa-Filho JM (2005). A review of natural products with antileishmanial activity.Phytomedicine. 12: 514-535.

26. Sadeghi-Nejad B, Shiravi F, Ghanbari S, Alinejadi M, Zarrin M (2010). Antifungal activity of Satureja khuzistanica (Jamzad) leaves extracts. Joundishapour JMicrobiol. 3:36-40.

27. Saedi Dezaki E, Mahmoudvand H, Sharififar F, Fallahi Sh, Monzote L, Ezatkhah F. (2015). Chemical composition along with anti-leishmanial and cytotoxic activity of Zataria multiflora. Pharm Biol. 8:1-7. 
28. Safarnavadeh T, Rastegarpanah M (2011). Anti-oxidants and infertility treatment, the role of Saturejakhuzestanica: a mini-systematic review. Iranian J. Reprod. Med. 9:61-70.

29. Sanchez de Rojas V, Somoza B, Ortega T, Villar AM, Tejerina T (1999). Vasodilatory effect in rat aorta of eriodictyol obtained from Satureja obovata. Planta Med. 65:234-238.

30. Signorini L, Gulletta M, Coppini D, Donzeli C, Stelini R, Manca L, Garosi R, Matteeli R (2007). Fatal disseminated toxoplasmosis during primary HIV infection. Curr HIV Res 5: 273-274.

31. Tavakoli Kareshk A, Keyhani A, Mahmoudvand H, Tavakoli Oliaei R, Asadi A, Andishmand M, Azzizian H, Babei Z, Ziali N (2015). Efficacy of the Bunium Persicum (Boiss) essential oil against acute toxoplasmosis in mice model. Iran J Parasitol. 10 (4): 625-631.

32. Ultee A, Bennik MHJ, Moezelaar R (2002). The phenolic hydroxyl group of carvacrol is essential for action against the food borne pathogen Bacillus cereus. Appl Environ Microbiol. 68:1561-1568.

33. Yesil Celiktas O, Hames Kocabas EE, Bedir E, Vardar Sukan F, Ozek T, Baser KHC (2007). Antimicrobial activities of methanol extracts and essential oils of Rosmarinus officinalis, depending on location and seasonal variations. Food Chem 100:553-559.

34. Zomorodian P, Ghadiri P, Saharkhiz MJ, Moein MR, Mehriar P, Bahrani F, Golzar T, Pakshir K, Fani MM (2015). Antimicrobial activity of seven essential oils from Iranian aromatic plants against common causes of oral infections. Jundishapur J Microbiol. 8(2): e17766. 